\title{
Consumption of Microwaved Food induces Oxidative Stress in the Liver and Brain of Sprague-Dawley Rats
}

\author{
${ }^{1}$ F. A Ajayi, ${ }^{2}$ R. O Afolabi,${ }^{3}$ C. N Chukwudozie, ${ }^{4}$ A.A Osuntoki. \\ ${ }^{1,2,3}$ Department of Biochemistry, College of Medicine of the University of Lagos, Idi-Araba, P.M. B. 12003, \\ Lagos, Nigeria.
}

\begin{abstract}
:
Introduction: Microwaves violently vibrate the water molecules in food, thus creating internal friction. The radiation, created by microwaves results in the destruction and deformation of food molecules, plus the formation of new radiolytic compounds..

Methods: In our present study, the susceptibility of microwave radiations on food fed to rats is studied by measuring antioxidant enzymes catalase, reduced glutathione and superoxide dismutase as well as measuring the level of TBARS (Thiobarbituric Acid Reacting Substances).

Results: It was observed from the result that the activities of antioxidant enzymes decreased drastically $(p<0.05)$ in rats fed the microwaved food as compared to their controls.

Conclusion: Microwave radiation affects the oxidative state of the liver and brain macromolecules. Damage caused by free radicals is aggravated by a reduction in protective antioxidant enzymes caused by the $M W$ interactions in biological tissues leads to production of ROS that eventually affect the status of the measured physiological parameters.

\section{Introduction}

Quite a number of people now make use of the microwave either for thawing or warming of food or even for fast cooking. As such, virtually every comfortable home now makes use of it. Advantages of microwave heating include savings in time and energy and easy to use. These advantages make it one of the most attractive cooking methods. Mechanism of microwave heating is different from the conventional heating. It represents the conversion of electromagnetic field energy to thermal energy, which arises in the field by polar water molecules in food (Sumnu, 2001). Bipolar water molecules are rotated in accordance with changes in the alternating electromagnetic field, which leads to the intermolecular friction and overcoming intermolecular forces, thus creating heat and leads to rapid heating of material. Microwave can penetrate to the depth of material, according to the dielectric parameters, thereby heating the material at the same time, both inside and on the surface. Free radicals are generated from many reactions in the body and can be created in foods from a combination of oxygen and light or heat that react with a donor molecule. Therefore, free radicals are not released, but are created. The main target or donor in foods is polyunsaturated fats because they have double bonds that accept oxygen and form a radical. Butter and margarine contain small amounts of polyunsaturated fats. For this reason, free radicals can theoretically occur in them when they are subjected to heat or light in the presence of oxygen. However, these foods also usually contain antioxidants which will reduce the free radicals. The risk of harm associated with microwaving butter or margarine from free radicals is, in my opinion, extremely low. (MacDonald, 2004). The unpleasant flavour of oxidized oils has been attributed to the secondary products of unsaturated fatty acids (Vieira \& Regitano-D'Arce 2001). The primary products of lipid oxidation are hydroperoxides that are generally referred to as peroxides.).
\end{abstract}

\section{Animal Protocol}

\section{Method of Study}

Twenty four white Sprague-Dawley rats (both male and female) were purchased from the Animal House of the College of Medicine, University of Lagos, Idi-Araba, Lagos State with body weight range of 108$188 \mathrm{~g}$. The animals were divided into four main groups (control groups 1 and 3 and the experimental groups 2 and 4 respectively). The formulated feed for the Control groups were not microwaved though with varying oil types. Microwaved feed however were fed to experimental groups 2 and 4.Animals in each group received standard (formulated) rat chow and water for six weeks as shown below: A total of 10kg Rat formulated feed was fed to the rats (fed with normal rat chow) and their weights were monitored. At the end of the respective experimental periods of $1,2,34,5$ and 6 weeks, the rats were sacrificed. 


\begin{tabular}{|l|l|}
\hline \multicolumn{1}{|c|}{ Nutrients } & \multicolumn{1}{c|}{$\begin{array}{c}\text { Composition } \\
(\mathrm{g})\end{array}$} \\
\hline Carbohydrate source (Rice) & 16.6 \\
\hline Protein source (Fish) & 1.6 \\
\hline Fat and Oil source (soyabean oil) & 0.8 \\
\hline Others (Vitamins, Minerals, Moisture) & 1 \\
\hline
\end{tabular}

- Group 1 - Control group with Animal fat (Not microwaved)

- Group 2 - Experimental group with Animal fat (Microwaved)

- Group 3 - Control group with Soya Oil (Not microwaved)

- Group 4 - Experimental group with Soya Oil (Microwaved)

\section{Sample Collection}

After twelve hours of fasting, $10 \mathrm{ml}$ of blood samples were collected from the rats by occular puncture and were sacrificed. The blood required for lipid and antioxidant assays was collected in lithium heparinized tubes. Blood samples were spun at $900 \mathrm{~g}$ and plasma was collected and stored in a bio-freezer at $-20^{\circ} \mathrm{C}$ until required for the biochemical assays.

\section{Assay method}

The antioxidants enzymatic activities assayed for are reduced glutathione, catalase and superoxide dismutase. The effect of the microwaved food on the rats was studied by measuring these enzymatic activities in the study groups in comparism with their respective control groups. The reduced glutathione (GSH) content of liver tissue as non-protein sulphydryls was estimated according to the method described by Sedlak and Lindsay (1968). Catalase activity was determined according to (Sinha, et.al.,1972). Superoxide Dismutase activity was determined according to (Sun and Zigma 1978). Lipid peroxidation marker, malondialdehyde was used to estimate the extent of peroxidation. This was determined using the method of (Buege and Aust 1978).

\section{Statistical analysis}

Numerical data were presented as mean values \pm standard deviation. The statistical analysis was carried out by one way ANOVA. The value of $\mathrm{p}<0.05$ was taken as statistically significant using GraphPad Prism 5 .

\section{Results}

Figures 1 and 4 showed that the control groups (1 and 3) had higher antioxidant enzymatic activities and malondialdehyde as compared with their respective study groups.

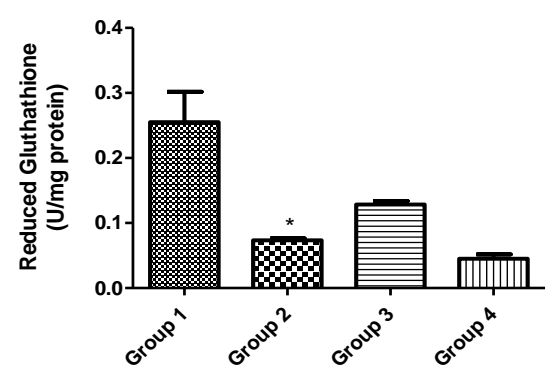

Figure 1: (a) Liver GSH

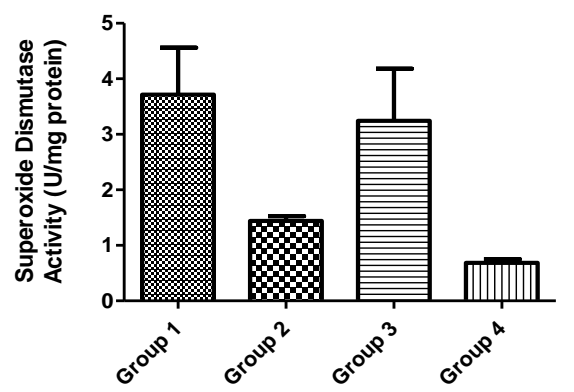

Figure 2: (a) Liver SOD

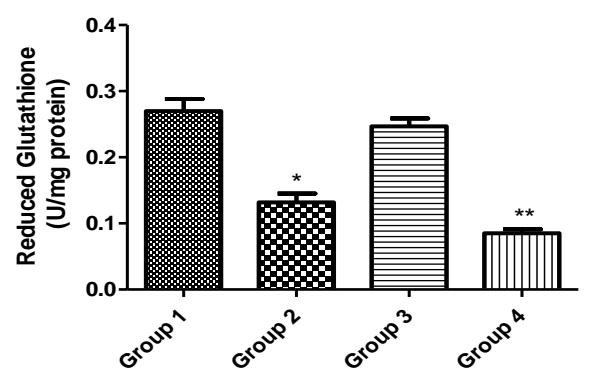

(b) Brain GSH

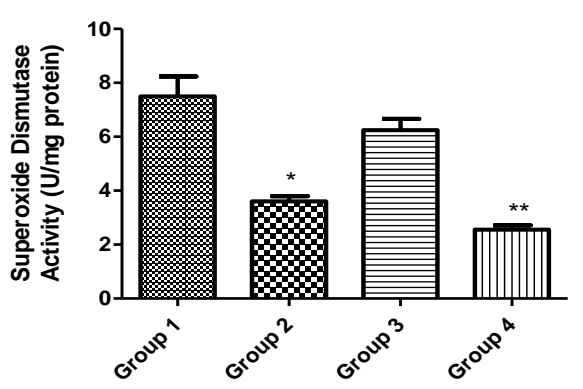




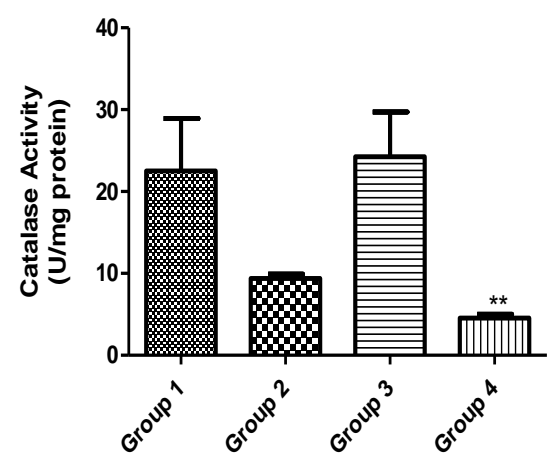

Figure 3: (a) Liver CAT

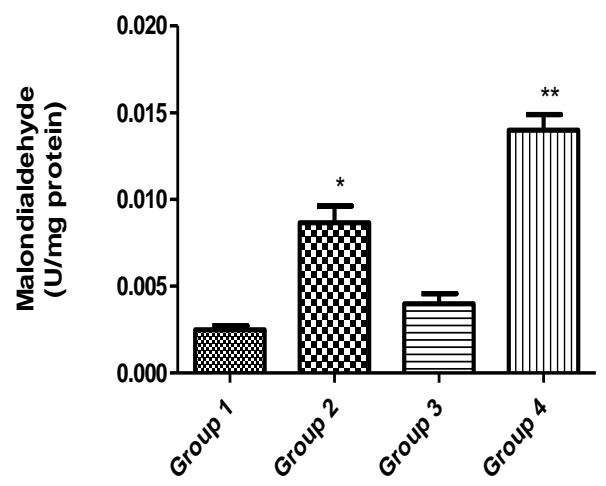

Figure 4: (a) Liver MDA

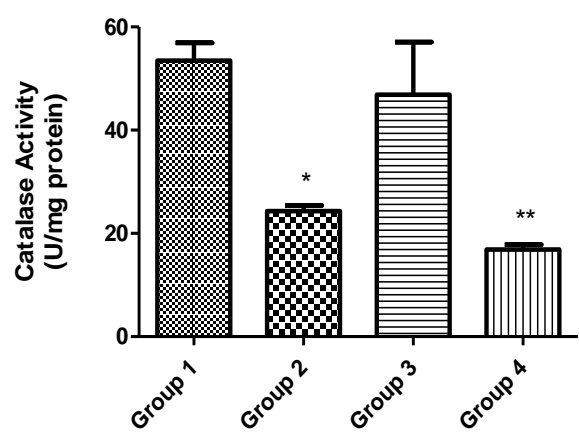

(b) Brain CAT

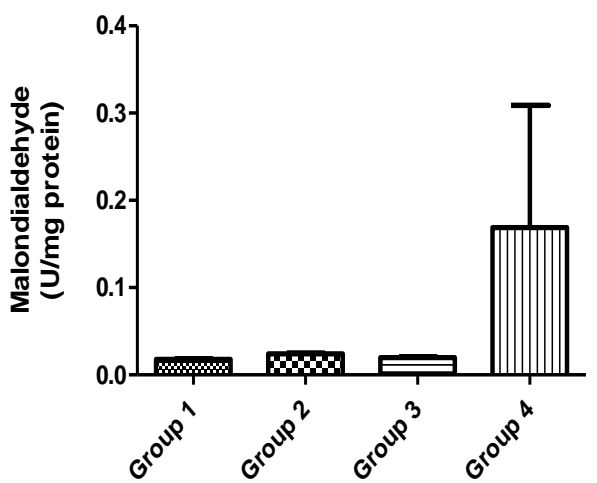

(b) Brain MDA

\section{Discussion}

The above results have shown that the activities of antioxidant enzymes reduced significantly at $\mathrm{P}<0.05$ both in the liver and brain of the microwaved-food fed rats in the (one and three respectively). When the level of antioxidants reduces, usually an increased level of pro-oxidants is observed which subsequently results in oxidative stress. As such, the ability of antioxidants to combat ravaging free radicals thereby stabilizing the system becomes defeated (Moustafa, 1998). It was observed also that as reduced glutathione depleted, there was more susceptibility of the study animals to free radicals-mediated damages. This is due to GSH's detoxifying role to free radicals deleterious effects (Hassan et al, 2004) including the damage induced by cellular lipid peroxidation. This observation is in line with some previous work (Fayun et al, 2007).

It is however needful to state that since antioxidants may be endogenously or exogenously produced, the ability of their system to produce antioxidants to bring a balance between the 'pro' and the 'anti' was overridden in the course of this study. This is due to the fact that the rats did not receive other food chows that are rich in antioxidants for the period of six weeks thus allowing for such a deleterious result. This may not likely be the case for humans who may get antioxidants from exogenous sources. This explains the drastic decline in antioxidant activities in these rats.

The extent of oxidative stress was also observed by measuring the lipid peroxidation marker, malondialdehyde (MDA). MDA was observed to significantly increase in the study groups 2 and 4 rats fed the microwave food. This results in cellular damage to organs and tissues allowing free radicals to invade the system. These results indicate that there is an association between the consumption of microwave food and the oxidative stress through distressing redox balance leading to physiological disturbances. It is therefore speculated that the changes induced by microwave irradiation may have cascade effect on the several biochemical processes. The observed increased level of lipid peroxidation, in this study is an indication to a high production of free radicals during the microwave exposure. These free radicals are highly oxidative moieties which directly affect the lipid membrane producing oxidative products such as GSSG and lipid peroxidation that increase also in many diseases and in tissues poisoned by a variety of toxins (Niedemhofer, 2003). The consequence of this however, is that the body system loses the ability to fight these radicals thus resulting in many diseases like artherosclerosis, Parkinson's disease, stoke e.t.c and in tissues poisoned by a variety of 
toxins (Gutteridge, 1993). Study rats of groups 2 and 4 fed with microwaved food were found to be more susceptible to lipid peroxidation,

Worthy of note also, is the fact that the mechanism of action of the microwave oven may be a reason for the resulting complications. The microwave oven emits two types of radiation; the microwaves or high frequency waves and the $60 \mathrm{~Hz}$ magnetic field. As such, molecules and cells hit by this hard electromagnetic radiation are forced to reverse polarity one to one hundred billion times a second. Atoms, molecules or cells of any organic system are usually not able to withstand such a violent destructive power, hence the microwaves violently vibrate the water molecules in food, thus creating internal friction, thereby heating the food from the inside out, while other forms of heat including the sun do not create friction heat in organic substances.

This implies that the radiation created by microwaves results in the destruction and deformation of food molecules, plus the formation of new radio lytic compounds, which are substances that are formed through the subjection to radiation. These compounds occur more when food is cooked with microwaves than in food that is cooked by conventional means.

\section{Conclusion}

The present study suggests that the microwave radiation affects the oxidative state of the liver and brain macromolecules. It has further been shown that the free radicals mediated damage is aggravated by a reduction in protective antioxidant enzymes caused by the MW interactions in biological tissues leads to production of ROS which cause oxidative reactions that eventually affect the status of the physiological parameters MDA and the activities of antioxidant enzymes. The possible health impacts of MW exposures continue to raise curiosities as more research works continue to reveal some proven facts through the use of experimental animals, human experiences and epidemiological studies.

\section{References}

[1]. Acworth, I.N. and B. Bailey. (1997). Reactive Oxygen Species, The handbook of oxidative metabolism. Massachusetts: ESA Inc., $1--4$.

[2]. Boothby, L.A. and P.L. Doering. (2005). Vitamin C and Vitamin E for Alzheimer's disease. Ann Pharmacother 12: 2073-2080

[3]. Botsoglou, N.A. et al. (1994). Rapid, Sensitive, and Specific Thiobarbituric Acid Method for Measuring Lipid Peroxidation in Animal Tissue, Food and Feedstuff Samples; J. Agric. Food chem., 42, 1931-1937.

[4]. Buege, J.A. and S.D. Aust. (1978). Microsomal lipid peroxidation methods. Enzymol., 52: 302-310.

[5]. Bull, A.W. and L.J. Marnett. (1985). Determination of Malondialdehyde by Ion-Pairing High-Performance Liquid Chromatography; Analyt. Biochem., 149, 284-290.

[6]. Fayun, L.I. et al. (2007). Hydroxyl radical gen eration and oxidative stress in carassius auratus liver. Chemosphere, 67: 13-19.

[7]. Georgieva, T. et al. (2002). Possibilities to control the health risk of petrochemical workers. Int. Arch. Occup. Environ. Health 75 S:21,S:26.

[8]. Gutteridge, J. (1993). Free radicals in disease processes: a compilation of cause and consequence. Free Radic. Res. Commun. 19, $141-158$.

[9]. Halliwell, B. (1992). Reactive oxygen species and the central nervous system. J. Neurochem. 59:1609-1623

[10]. Halliwell, B. (2007). Biochemistry of oxidative stress. Biochemical Society Transactions, 35, 1147-1150.

[11]. Halliwell, B. and S. Chirico. (1993). Lipid peroxidation: Its mechanism, measurement, and signficance. Am. J. Clin. Nutr., 57: S715-S725.

[12]. Hassan, N. S., Ramadan A. S., Aziz S. W., and A. M. Attia. (2004). Effect of different magnetic field intensities on bloodative stress, J. Genetic Eng. \& Biotechnol. (NRC), 2: 63-72.

[13]. Imlay, J. A. (2003). Pathways of oxidative damage. Annu. Rev. Microbiol. 57: 395-418.

[14]. Kanazawa, A., et al. (2002). Dietary lipid peroxidation products and DNA damage in colon carcinogenesis. Eur. J. Lipid Sci. Technol. 104, 439-447.

[15]. Moustafa, S.(1998). Effects of glutathione depletion on carbohydrate metabolism in the rat, Commun. Pharmacol. Toxicol. 3: 5564.

[16]. Nathan, C. and M.U. Shiloh. (2000). Reactive oxygen and nitrogen intermediates in the relationship between mammalian hosts and microbial pathogens. Proc. Natl. Acad. Sci. U.S.A. 97 (16): 8841-8848

[17]. Naz, S. et al. (2004). Oxidative stability of olive, corn and soybean oil under different conditions. Food Chemistry, 88: $253-259$.

[18]. Naziroglu, M. et al. (2004). Selenium and high dose vitamin E administration protects cisplatin-induced oxidative damage to renal, liver and lens tissues in rats. Toxicology, 195: 221-230.

[19]. Niedemhofer L. J., Daniels, J.S., Rouzer C.A., and R.E Greene. (2003). Malondialdehyde, a product of lipid peroxidation is mutagenic in Human cells. J Biol Chem, 278; 31426-31433.

[20]. Nijs, J. et al. (2006). Chronic musculoskeletal pain in chronic fatigue syndrome: recent developments and therapeutic implications. Man Ther 11 (3): 187-191.

[21]. Sedlak, J. and R. H. Lindsay. (1968). Estimation of total, protein-bound and non-protein sulfhydryl groups in tissue with Ellman's reagent. Anal. Biochem. 25: 192-205

[22]. Sinha, A.K, (1972). Colorimetric Assay of catalase. Anal. Biochem. 47. 2: 389-394.

[23]. Sumnu, G., (2001). A review on microwave baking of foods. INT. J. Food Sci. Technol., 36: 117-127.

[24]. Sun, M. And Zigma, S., (1978). An improved spectrophotometer assay of superoxide dismutase based on Epinephrine Antioxidation. Analytical Biochemistry. 90: 81-89. 